\title{
Multivariate predictive models for the prediction of fatty acids in the EU high added-value "acorn Iberian pig ham" using a miniature near-infrared spectroscopy instrument
}

\author{
Ana Garrido-Varo, Cecilia Riccioli, Tom Fearn, E. De \\ Pedro, Dolores C. Pérez-Marín
}

Ana Garrido-Varo, Cecilia Riccioli, Tom Fearn, E. De Pedro, Dolores C. Pérez-Marín, "Multivariate predictive models for the prediction of fatty acids in the EU high added-value "acorn Iberian pig ham" using a miniature nearinfrared spectroscopy instrument," Proc. SPIE 11016, Sensing for Agriculture and Food Quality and Safety XI, 110160C (30 April 2019); doi: 10.1117/12.2521977

Event: SPIE Defense + Commercial Sensing, 2019, Baltimore, Maryland, United States 


\title{
Multivariate predictive models for the prediction of fatty acids in the EU high added-value "acorn Iberian pig ham" using a miniature near- infrared spectroscopy instrument.
}

\author{
Ana Garrido-Varo ${ }^{1 *}$, Cecilia Riccioli ${ }^{1}$, Tom Fearn ${ }^{2}$, E. De Pedro ${ }^{1}$ and Dolores Pérez- Marín ${ }^{1}$ \\ ${ }^{1}$ Non- Destructive Spectral Sensors Unit. Faculty of Agricultural and Forestry Engineering, \\ University of Córdoba, Córdoba, Spain *pa1gavaa@uco.es \\ ${ }^{2}$ Department of Statistical Science,University College London, UK.
}

\begin{abstract}
Acorn Iberian ham (Jamón Ibérico de Bellota) is one of the most expensive luxury foodstuffs produced in Europe, with a highly appreciated smell and flavour. Its recognized high-sensorial quality and health properties are mainly due to the traditional outdoor feeding system (Montanera) of Iberian pigs (IP), which provides high standards of animal welfare. Nowadays, one of the frauds affecting this product is the use of "special compound feeds" to simulate the fat composition of the acorns through the inclusion of sources of oleic acid like the ones found in pigs fed outdoors. The high prices paid for a cured leg of Iberian ham -ranging from hundreds to thousands of euros- leads to many opportunities for mislabelling and fraud. Fatty acid content of the adipose tissue could provide evidence of the feeding system. Gas chromatography (GC) is used at industry level for production control purposes. However, it is costly and time-consuming, and it is only applied to batches of animals rather than individual pigs. The main goal of this study was to use spectra belonging to a portable NIRS instrument (MicroNIR Onsite Lite, Viavi Solutions Inc.) for on-site quantitative (fatty acid content) analysis of individual Iberian pork carcasses at the slaughterhouse. Performance of this portable instrument was compared with an at-line NIRS monochromator. PLS models were built and optimized resulting in standard errors of cross validation ranging from 0.83 to 0.84 for palmitic acid, 0.94 to 0.99 for stearic acid, 1.47 to 1.56 for oleic acid and 0.53 to 0.58 for linoleic acid.
\end{abstract}

Keywords: NIRS, in situ quality control, fatty acids, quantitative models, Iberian ham, high-value food products.

\section{INTRODUCTION}

The determination of the fatty acid (FA) profile has a high relevance for the quality control of Iberian pig (IP) meat products. FA profile of the subcutaneous adipose tissue performed by gas chromatography (GC) has been traditionally used for classifying and authenticating animals in different commercial categories. Furthermore, nowadays, due to the consumer demands and legal requirements, ${ }^{1}$ the information displayed in the food label for local, traditional and added value meat products is very important. Near Infrared Reflectance Spectroscopy (NIRS) has shown its potential for predicting FA composition in IP melted fats, adipose and meat tissues with a variable grade of precision and accuracy. ${ }^{23}$ Garrido-Varo, García-Olmo and Pérez-Marín ${ }^{4}$ indicated that the differences found in the estimation of the main fatty acids and its overall statistical performance depend on the sample presentation, analysis mode and instrument characteristics. Fat analysis by NIRS can be useful as a substitute for GC analysis to predict the main fatty acid composition, specially, palmitic acid (C16:0, stearic acid (C18:0), oleic acid (C18:1) and linoleic acid (C18:2). Moreover, the industry is interested in simplification of the sampling/sample presentation to perform individual analysis at the speed of the slaughter process and in the use of new low-cost NIRS instrumentation.

NIRS has been studied for these purposes based on fibre-optic instrumentations showing potential for the prediction of oleic, linoleic, palmitic and stearic fatty acids. ${ }^{5}$ As in other food products, the variability between campaign years is a complex task that can affect the suitability of the technology. In extensive IP productions, the lack of uniformity in the

Sensing for Agriculture and Food Quality and Safety XI, edited by Moon S. Kim, Bryan A. Chin, Byoung-Kwan Cho, Proc. of SPIE Vol. 11016, 110160C - @ 2019 SPIE · CCC code: 0277-786X/19/\$18 · doi: 10.1117/12.2521977 
chemical composition between individual animals and different slaughtering campaigns, due probably to variability in the diet based on natural resources of the field, has been shown. A further step, rather than take the sample from the process and present it to the instrument, is to move the instrument to the process line, making non-destructive in-situ NIRS analysis a reality.

In this study, different instruments are evaluated for the quantitative at-line and in situ fatty acid profile prediction (C16:0, C18:0, C18:1 and C18:2) in adipose tissues using the same dataset. The study compares the performance and accuracy of the at-line application versus an in situ application based on a handheld device implemented at the carcass process line.

\subsection{Sample set}

\section{MATERIAL AND METHODS}

The data set comprises 495 samples from 45 different producers. The samples were measured in two years: 66 were measured in 2016 and the remaining 429 in 2017. Just over half (265) were premium grade (bellota).

\subsection{Fatty acids measurements}

Table 1 below presents summary statistics for the distribution of the reference measurements of the fatty acids in all 495 samples.

Table 1. Summary statistics for fatty acids in 495 samples

\begin{tabular}{l|l|l|l|l}
\hline & Mean (\%) & SD (\%) & Min (\%) & Max (\%) \\
\hline C16:0 & 23.4 & 2.1 & 18.4 & 28.9 \\
\hline C18:0 & 12.0 & 2.3 & 7.7 & 18.6 \\
\hline C18:1 & 50.1 & 3.7 & 40.9 & 58.3 \\
\hline C18:2 & 8.0 & 1.1 & 4.8 & 11.4 \\
\hline
\end{tabular}

\subsection{Spectral measurements}

Samples were measured using both a FOSS NIRSystems 6500 and a MicroNIR 1700. Before the FOSS spectra were used to develop calibrations, they were cut to the MicroNIR range (908-1676nm) and interpolated using cubic splines to give absorbances at the same 125 wavelength points as the MicroNIR. Before the interpolation, the step in the FOSS spectra at $1100 \mathrm{~nm}$ resulting from the change of detectors was removed by simply shifting the spectrum below $1100 \mathrm{~nm}$ upwards to make a smooth join.

Figure 1 shows the mean spectra of the whole population for the two NIR instruments used. 


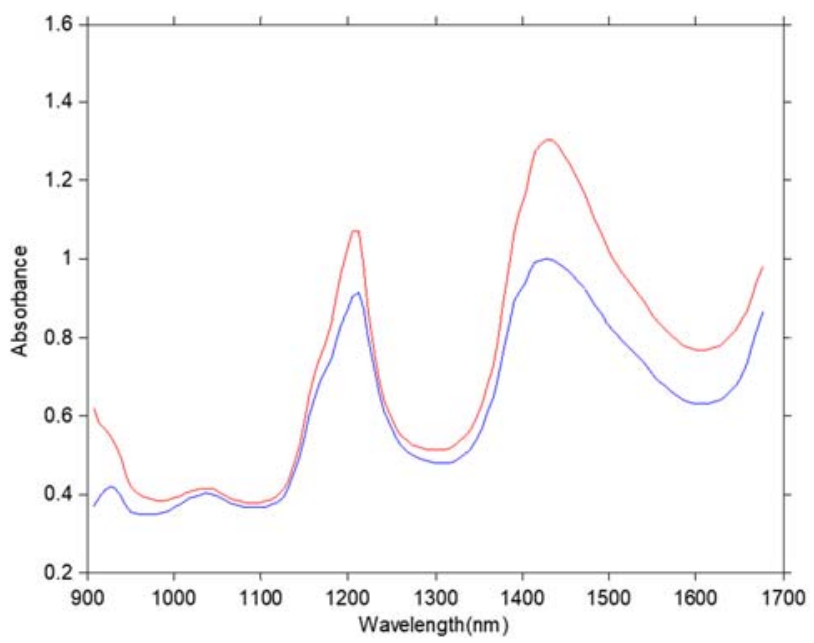

Figure 1. Mean spectra from FOSS (blue) and MicroNIR (red).

\subsection{Data Processing}

Different software and chemometric packages were used for data processing. The prediction algorithms were developed using the MATLAB R2018a software package (The Mathworks, Natick, USA). All the calibrations were assessed by a cross-validation leaving out each of the 45 producers in turn. The pre-treatment and the PLS calibrations were carried out using the PLS Toolbox v8.21 (Eigenvector Research, USA).

Partial Least Square (PLS) regression ${ }^{6}$ were used for calibration development of the FA models. The optimum number of PLS factors was selected by cross-validation using venetian blinds. For the calibrations with both the Foss and the microNIR instruments, six pre-treatments were investigated: raw absorbance spectra, first derivative, and second derivative, each tried without and with SNV. In the case of two treatments, the SNV was applied after the derivative.

\section{RESULTS AND DISCUSSIONS}

The first step was to seek calibrations for the 4 fatty acids using 125-point FOSS spectra. This sets a target or what one might hope to achieve when using a scientific grade equipment designed to be used at well controlled laboratory conditions. Among all the calibrations developed using the pre-treatments previously descripted, the equations that showed better accuracy and precision were the ones obtained using second derivative, calculated by a Savitzky-Golay filter with a second order polynomial and a widow width of 5 points, which is around $30 \mathrm{~nm}$ with these 125-point spectra, and then SNV.

The RMSECV values, using leave-out-one procedure and numbers of factors are given in Table 2, and plots of observed values versus cross-validated predictions are given in Figure 2. 
Table 2. Numbers of PLS factors and root mean squared error of cross-validation (RMSECV) for separate PLS calibrations for four fatty acids using 125-point FOSS spectra.

\begin{tabular}{l|l|l}
\hline Fatty acid & Factors & RMSECV (\%) \\
\hline C16:0 & 8 & 0.63 \\
\hline C18:0 & 6 & 0.76 \\
\hline C18:1 & 8 & 1.10 \\
\hline C18:2 & 6 & 0.47 \\
\hline
\end{tabular}


Figure 2. Cross-validated predictions versus reference values for C16:0 (up left), C18:0 (up right), C18:1 (bottom left) and C18:2 (bottom right), Foss spectra

All these plots show some outliers, and other diagnostic plots also show problems with a small number of samples. With some data removed the results might be expected to improve a little, but with 495 samples and nearly all of them good, the improvement will be modest, and for the moment all samples have been left in.

Using the MicroNIR spectra with the same pre-treatment as the FOSS (second derivative+SNV) gave the results in Table 3 . 
Table 3. Numbers of PLS factors and root mean squared error of cross-validation (RMSECV) for separate PLS calibrations for four fatty acids using MicroNIR spectra

\begin{tabular}{l|c|c}
\hline Fatty acid & Factors & RMSECV (\%) \\
\hline C16:0 & 14 & 0.84 \\
\hline C18:0 & 4 & 0.94 \\
\hline C18:1 & 13 & 1.47 \\
\hline C18:2 & 13 & 0.58 \\
\hline
\end{tabular}

Plots of observed values versus cross-validated predictions are given in Figure 3.
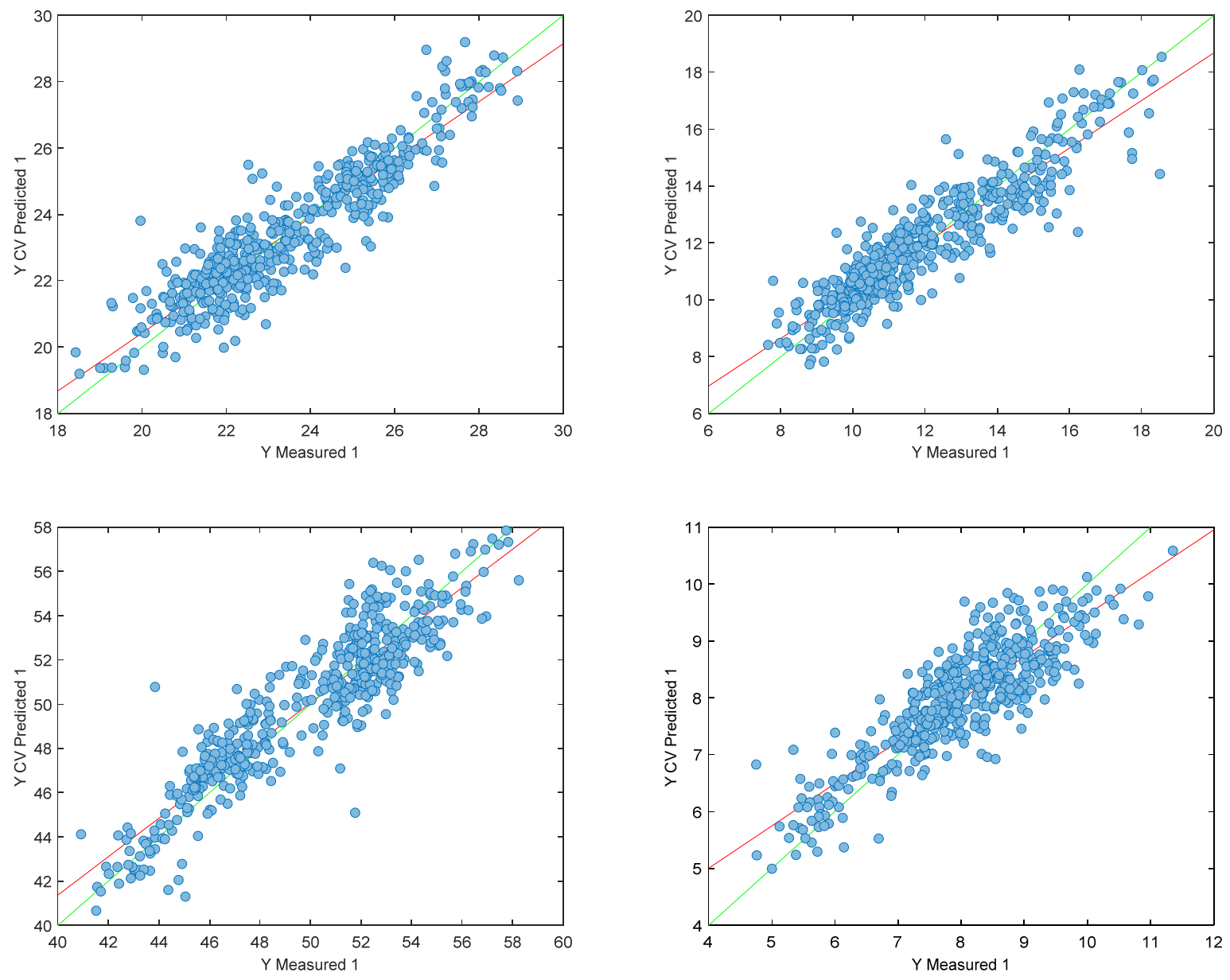

Figure 3. Cross-validated predictions versus reference values for C16 (up left), C18 (up right), C18:1 (bottom left) and C18:2 (bottom right), MicroNIR spectra.

All four calibrations are visually worse with the MicroNIR than with the FOSS. The RMSECV values are consistently $20-30 \%$ worse with the MicroNIR. 


\section{DISCUSSION, CONCLUSIONS AND FURTHER WORK}

As expected, the performance of the portable device is lowest that for the high-grade scientific instrument. However, there are several different advantages is using a handheld instrument which are worth to consider like the low cost or the fact that samples don't need to be collected and sent to the lab. But, most importantly, the portable instrument can be used to analyse every single pig carcass at the slaughterhouse. The significative decrease in the sampling error that can be reached with the microNIR outweighs the loss of precision of the predictive models. Furthermore, nonlinear regression approaches will be tried, though first some of the obvious outliers need to be removed. Also, various approaches to leveraging the historical FOSS data will be explored.

\section{REFERENCES}

[1] EU., "Position (EU) No 7/2011 of the Council at first reading with a view to the adoption of a Regulation of the European Parliament and of the Council on the provision of food information to consumers, amending Regulations (EC)No 1924/2006 and (EC) No 1925/200,” Off. J. Eur. Union(54), 1-59.

[2] Fernández-Cabanás, V. M., Garrido-Varo, A., Olmo, J. G., Pedro, E. De and Dardenne, P., "Optimisation of the spectral pre-treatments used for Iberian pig fat NIR calibrations," Chemom. Intell. Lab. Syst. 87(1), 130-138 (2007).

[3] Pérez-Marín, D., Garrido-Varo, A., De Pedro, E. and Guerrero-Ginel, J. E. E., "Chemometric utilities to achieve robustness in liquid NIRS calibrations: Application to pig fat analysis," Chemom. Intell. Lab. Syst. 87(2), 241246 (2007).

[4] Garrido-Varo, A., García-Olmo, J., Pérez-Marín, D., "Analysis of fats and oils," [Near Infrared Spectroscopy in agriculture], J. B. Roberts C.A., Workman J., Reyes III, Ed., American Society of Agronomy, Crop Science Society of America, Soil Science Society of America, Madison, Wisconsin, USA, 487-558 (2004).

[5] Pérez-Juan, M., Afseth, N. K., González, J., Díaz, I., Gispert, M., Furnols, M. F. i, Oliver, M. A. and Realini, C. E., "Prediction of fatty acid composition using a NIRS fibre optics probe at two different locations of ham subcutaneous fat," 1416-1422 (2010).

[6] Windham, W. R., Mertens, D. R. and Barton, F. E., "Protocol for NIRS calibration: sample selection and equation development and validation," [Near Infrared spectroscopy (NIRS): Analysis of Forage Quality. Agriculture Handbook], J. S. Martens, G.C., Shenk, Ed., USDA-ARS, US Government Printing Office, Washington D.C., 96-103 (1989). 